Instituto Internacional de Investigación y Desarrollo Tecnológico Educativo INDTEC, C.A.

DOI: https://doi.org/10.29394/Scientific.issn.2542-2987.2021.6.20.19.340-359

OAl-PMH: http://www.indteca.com/ojs/index.php/Revista Scientific/oai

Ensayo Original / Original Essay

\title{
La Interdisciplinariedad desde la Perspectiva de Ezequiel Ander-Egg
}

\author{
Autora: Lisbeth Adays Rengifo Avadez \\ Universidad Nacional Experimental Politécnica de la Fuerza Armada, UNEFA \\ lisbethrengifo@gmail.com \\ Los Teques, Venezuela \\ https://orcid.org/0000-0002-6153-9769
}

\section{Resumen}

El sistema educativo se ha estado accionando en consonancia al paradigma tradicional, caracterizado por seccionar, sesgar, atomizar el todo en las unidades de estudio que le conforman; de ahí que surge la disciplinariedad, que delimita las diferentes áreas del conocimiento de manera tal que se encuentran organizados de con una postura sistemática para facilitar su comprensión; sin embargo, Ander-Egg (1999), le dio un giro al exponer respecto una visión de ejecutar la interdisciplinariedad manifestando que las líneas interpuestas entre cada disciplina están difusas y que al encontrarse imbricadas pueden aportar soluciones a problemas complejos del entorno educativo, el trabajo en equipo, ampliar la visión de la realidad considerando las distintas perspectivas de cada profesional bajo la visión de complementariedad. El propósito de este ensayo es analizar la interdisciplinariedad en educación desde la visión del autor. El análisis de contenido se realizó a través de textos de investigadores como Morin (2007); Balza (2001); Bertalanffy (1968); Prigogine y Stengers (1990), entre otros, acerca de interdisciplinariedad, complejidad y enfoque sistémico. La conclusión más resaltante es que se pueden incorporar cambios educativos a nivel institucional, incorporando la interdisciplinariedad en su accionar.

Palabras clave: interdisciplinariedad; complejidad; enfoque sistémico. Código de clasificación internacional: 5802.04 - Niveles y temas de educación.

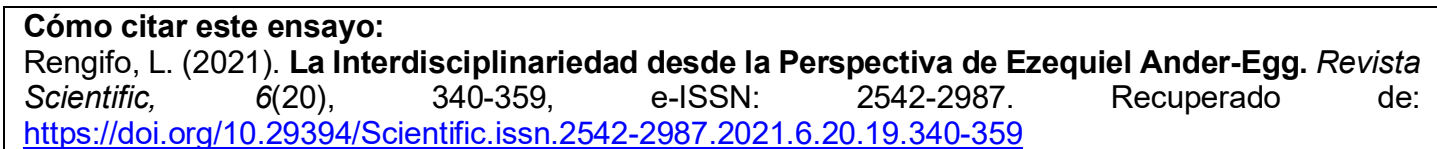

Fecha de Recepción: 08-01-2021
Fecha de Aceptación:

31-03-2021
Fecha de Publicación: 05-05-2021 


\title{
Interdisciplinarity from the Perspective of Ezequiel Ander-Egg
}

\begin{abstract}
The educational system has been operating in accordance with the traditional paradigm, characterized by sectioning, skewing, atomizing the whole in the study units that comprise it; Hence the disciplinarity arises, which delimits the different areas of knowledge in such a way that they are organized in a systematic position to facilitate their understanding; However, Ander-Egg (1999), gave it a twist by exposing a vision of executing interdisciplinarity, stating that the interposed lines between each discipline are diffuse and that when they are overlapped they can provide solutions to complex problems in the educational environment, work As a team, broaden the vision of reality considering the different perspectives of each professional under the vision of complementarity. The purpose of this essay is to analyze interdisciplinarity in education from the author's point of view. The content analysis was carried out through texts by researchers such as Morin (2007); Balza (2001); Bertalanffy (1968); Prigogine and Stengers (1990), among others, about interdisciplinarity, complexity and a systemic approach. The most striking conclusion is that educational changes can be incorporated at the institutional level, incorporating interdisciplinarity in their actions.
\end{abstract}

Keywords: interdisciplinarity; complexity; systemic approach. International classification code: 5802.04 - Levels and subjects of education.

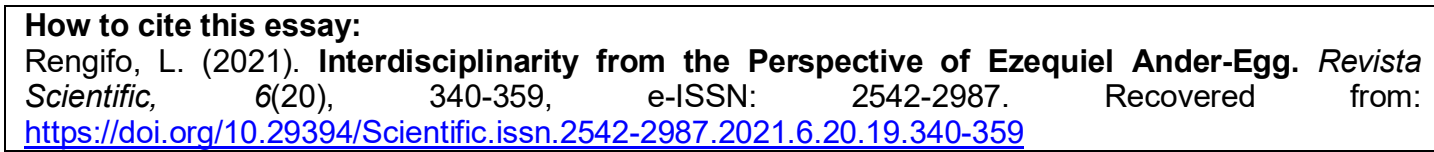

Date Received: 08-01-2021
Date Acceptance: 31-03-2021
Date Publication: 05-05-2021 


\section{Introducción}

Las instituciones educativas han estado transitando basadas en un pensamiento lineal, de causa efecto, cónsono con el diseño del sistema en el que prevalece la disciplina como epicentro en el cual se desarrollan todas las actividades inherentes en educación. De aquí que se hace necesario realizar un cambio de visión de la realidad hacia la interdisciplinariedad.

Se considera relevante analizar La Interdisciplinariedad en Educación, desde la óptica de Ander-Egg (1999a): autor con un pensamiento holístico, respetuoso de la disciplinariedad como eje necesario para consolidar la interdisciplinariedad.

Es importante indicar que la interdisciplinariedad se desarrolla bajo el enfoque de la complejidad, término que apunta a pasar de un extremo a otro, desde una realidad sesgada a una con diferentes puntos de vista que enriquezcan y complementen el enfoque causa efecto. La educación está siendo estudiada desde lo interdisciplinario para favorecer cambios desde su funcionamiento que implica ver más allá de lo disciplinario, cónsono con las demandas sociales.

El propósito de este ensayo fue examinar el punto de vista de diversos autores en relación con el enfoque que se tiene de la interdisciplinariedad y su implicación en la educación y cómo a través de ella se puede realizar transformación de la praxis docente.

En este orden de ideas se realizó un arqueo bibliográfico para abordar los términos propios para la realización de este ensayo y sobre los que se dirigen sus diferentes estadios, analizar y comprender la información para establecer posturas respecto a la temática.

\section{Desarrollo}

\subsection{La Interdisciplinariedad en Educación}

El profesor Ander-Egg (1999b): como pedagogo, ensayista y 
epistemólogo ha escrito libros en sus diversas áreas de conocimiento: Sociología, Ciencias Políticas, Economía, Planificación Económica, Planificación Social y Pedagogía, Doctor en Ciencias Políticas y Económicas, además de ser conferencista en encuentros internacionales, seminarios, congresos y talleres. Uno de sus intereses es estudiar la transformación de la sociedad y la falta de adaptación de las instituciones para dar respuestas a la problemática mundial.

Su interés como investigador es la realización de estudios de trabajo social, dejando aportes en la investigación de técnicas de desarrollo social. Su postura epistemológica está basada en el paradigma socio crítico, que profesa el cambio en contextos sociales con la participación de quienes viven en realidades problematizadas.

Respecto al libro de Ander-Egg (1999c): titulado La Interdisciplinariedad en Educación, el autor realiza una investigación de cómo se plasma la interdisciplinariedad en la educación. La obra se divide en dos capítulos. Cada capítulo ofrece de manera didáctica una introducción, desarrollo de ideas principales y secundarias, para luego cerrar con una conclusión. El escritor inicia introduciendo al lector en un nuevo vocabulario para hacer más comprensible la lectura del libro.

Se transita en una reflexión respecto a la interdisciplinariedad en la que explica, el léxico cónsono con esta área, así como el origen de la especialización disciplinar. Luego describe el enfoque, la actitud intelectual y existencial de la interdisciplinariedad hasta llegar a su operacionalización en la educación.

Del texto se resalta la fluidez del lenguaje, sencillo y lo suficientemente técnico, los títulos y subtítulos están concatenados de manera tal que es de fácil comprensión. Constituye un ensayo producto de su participación como ponente en unas jornadas de investigación. La escritura es reflexiva, considerando al lector en una postura de investigador y cuestionador de las 
Instituto Internacional de Investigación y Desarrollo Tecnológico Educativo INDTEC, C.A.

DOI: https://doi.org/10.29394/Scientific.issn.2542-2987.2021.6.20.19.340-359

OAI-PMH: http://www.indteca.com/ojs/index.php/Revista Scientific/oai

Ensayo Original / Original Essay

realidades hasta ahora exploradas y que pueden repensarse para abrir nuevos modelos de pensamiento que permitan un nuevo abordaje de indagar realidades sociales a través de la educación valiéndose de la interdisciplinariedad.

\subsection{Análisis de la Construcción de Interdisciplinariedad de Ezequiel Ander-Egg}

Se reconoce, que existe una interpretación errónea por parte de los educadores, esto producto de cómo se ha estructurado y se ha puesto en praxis el sistema educativo, la educación por objetivos. Debido a esto, el autor menciona y conceptualiza términos con los que se tiende a confundir la interdisciplinariedad. Paralelamente, Ander-Egg (1999d), expone aceptando que existe un modismo respecto al término de interdisciplinariedad, definiéndola como:

Interprofesionalidad: [...] profesionales de diferentes campos que trabajan juntos sobre un mismo objeto o sobre un mismo problema; Multidisciplinariedad: [...] no hay interpenetración de unas ciencias con otras [...]; Disciplinariedad Cruzada: [...] se produce cuando la problemática de una disciplina trasciende a otra u otras [...]; Transdisciplinariedad: [...] una instancia científica capaz de imponer su autoridad a las disciplinas particulares [...]; Interdisciplinariedad: [...] es la idea de interacción y cruzamiento entre disciplinas y en orden a la comunicación de conocimientos (págs. 27-31).

Indudablemente que en la realidad existe un abordaje de la realidad considerando desde la óptica de cada profesional de aportar análisis de un objeto de conocimiento con una yuxtaposición de elementos aportados con la convicción de que se está realizando interdisciplinariedad.

La interdisciplinariedad implica hacer un cambio de pensamiento lineal y estructurado, en el que se da cabida al abordaje de realidades desde una sola perspectiva, desde el pensamiento disciplinar, involucra apertura mental 
Instituto Internacional de Investigación y Desarrollo Tecnológico Educativo INDTEC, C.A.

DOI: https://doi.org/10.29394/Scientific.issn.2542-2987.2021.6.20.19.340-359

OAI-PMH: http://www.indteca.com/ojs/index.php/Revista Scientific/oai

Ensayo Original / Original Essay

para visualizar escenarios de estudio desde diferentes ángulos, con la participación de diferentes profesionales que enriquezcan la investigación. Para ello es importante asumir el paradigma de la complejidad que parte del principio de tener una visión micro de la realidad y holística de la misma, sin perder de vista las interacciones que tienen los elementos involucrados en el fenómeno de estudio. La interdisciplinariedad es conceptualizada por Martínez (1997a), como:

La interdisciplinariedad es un viejo concepto que se basa en valores muy aceptados, como la integración, la síntesis y la unidad del conocimiento [...] es también parte de una evolución general del conocimiento, que emerge a través de un proceso tanto de diferenciación -división de un objeto en nuevas y más pequeñas especialidades- como de fusión de perspectivas separadas en relaciones comunes $y$, a veces, nuevas. Finalmente, funciona como un medio de solucionar problemas "prácticos" dentro de la sociedad. Aunque algunas actividades interdisciplinarias pueden ser muy diferentes de otras, todo el trabajo de esta naturaleza se apoya en una epistemología fundamental de convergencia, en una síntesis integradora que tiende a producir diferentes tipos de interacción disciplinaria (pág. 110).

La interdisciplinariedad por lo tanto no niega la existencia de las disciplinas, por el contrario, las reconoce y las resalta, de manera que cuando por separado no aportan soluciones, entonces es cuando debe surgir esta nueva corriente de pensamiento. Implica la integración de saberes, interpretando realidades desde la unidad disciplinar hasta alcanzar la totalidad, con un enfoque dialéctico.

Es indispensable que cada profesional tenga las competencias y conocimientos en cada una de su área de conocimiento, debe existir por lo tanto un punto de convergencia en el discurso, por lo cual se trasciende para generar un novedoso proceso lingüístico. Conforme a lo expresado por Balza (2011), el concepto de interdisciplinariedad, son todas aquellas: 
[...] Conexiones cognoscitivas interdisciplinarias desde la ontología de los procesos de investigación, normalmente se manifiestan a través de los nexos entre profesionales, campos particulares del conocimiento, perspectivas paradigmáticas y transferencias de metodologías y técnicas con el objeto de integrar aportes y contenidos, que al imbricarlos de modo concurrente orientan la solución de aquellas problemáticas que son objeto de investigación (pág. 82).

Los hechos históricos, remontado desde la época medieval en la que el conocimiento se fragmentó debido al auge y creciente acumulación de saberes; posteriormente la ciencia durante y después del Renacimiento se acondicionó para avanzar y desarrollarse, así como la tecnología, transformando el mundo en cuatro siglos. Debate que actualmente la ciencia y los paradigmas de pensamiento urgen de la integración de las disciplinas en el abordaje de la realidad, para Ander-Egg (1999e): hoy la preocupación y búsqueda por la articulación de los saberes y la integración de conocimientos, tiene lugar en diferentes ciencias, aún como exigencia del desarrollo de las mimas ciencias.

Respecto al ámbito educativo Ander-Egg (1999f): arguye que el gran desafío es captar la totalidad no dividida lo que supone un modelo de enseñanza aprendizaje que establezca conexiones y relaciones de los saberes en una totalidad no dividida y en permanente cambio, bajo un enfoque globalizador e interdisciplinario, considerando la organización y articulación de los conocimientos de cada disciplina. Significa la integración de disciplinas que hasta el momento están desunidas producto de los cambios que surgieron en la manera de hacer ciencia. Es decir, la nueva teoría debe unir los conocimientos generados por cada disciplina.

Así mismo, el autor afirma que en función a los cambios que se vienen generando en las ciencias, el sistema educativo no puede estar al margen de ello, por lo cual debe transformarse en función a la demanda social, a las 


\section{Ensayo Original / Original Essay}

complejidades propias de contextos que se caracterizan por ser distópicos, con problemas diversos que pueden ser solventados desde la apreciación de diversas fuentes, con ópticas distintas.

La transformación debe darse bajo un marco organizador que el autor reseña: a). debe partir de la racionalidad, extrapolación y formalismo de las matemáticas; b). a través de un enfoque sistémico concibiendo el sistema como un todo; c). noción de estructura como dimensión subyacente que permita entender las relaciones necesarias no observables que existen en toda realidad en términos operacionales; d). parte de la lógica de la complejidad: asociación de lo que está desunido y concebir la multidimensionalidad de la realidad. En este sentido es oportuno considerar la concepción que tiene Morín, citado por Ander-Egg (1999g), de la complejidad:

El problema de la complejidad debe plantarse correlativamente en el marco gnoseológico (el pensamiento de la realidad) y en el marco ontológico (la naturaleza de la realidad). Es decir, que la complejidad concierne a la vez a los fenómenos, a los principios fundamentales que rigen a los fenómenos, a los principios fundamentales -metodológicos, lógicos, epistemológicos- que rigen y controlan nuestro pensamiento (pág. 60).

El estudio de los fenómenos desde el paradigma de la complejidad amerita un cambio de configuración de pensamiento de quienes hacen ciencia, desde su epistemología, el cómo se hace el abordaje del objeto de estudio y la relación del sujeto objeto, es decir, debe repensarse la forma en que se está haciendo ciencia, abrirse paso a un nuevo pensamiento. En este sentido, Prigogine y Stengers (1990), afirman respecto a la complejidad:

El descubrimiento de la complejidad es, ante todo, un desafío. Nos recuerda que nuestras ciencias están todavía dando sus primeros pasos, que fueron apasionadas pero a veces presuntuosas. Hoy empezamos a reconocer lo que implica la idea de un mundo intrínsecamente activo $y$, por tanto, a comprender hasta dónde llega nuestra ignorancia. Pero esta 
complejidad lleva también consigo la esperanza de una nueva identidad de la ciencia [...] las ciencias no tienen, por derecho, otro límite que el de la creatividad humana (págs. 352-353).

Los autores reseñados refieren la importancia de mirar las ciencias desde una visión diferente a la física, su lógica ha sido aplicable a otras esferas del saber, por lo que instan a hacer un viraje, un redescubrimiento, debe hacerse generalizable a otros campos del saber, es necesario un nuevo diálogo entre las ciencias, considerando el hombre y su cultura.

La manera de hacer ciencia en contextos sociales no puede ser igual al abordaje que se puede realizar en la naturaleza, ya que el hombre es complejo, como ser biológico, psicológico, emocional y social. La cuantificación de los hechos para estudiarlos desde la concepción lineal, de causa efecto se ha ido modificando para construir un pensamiento complejo. En el desarrollo de las ciencias complejas ha surgido una nueva configuración y Ander-Egg (1999h): las refleja y denomina como las metáforas propias del paradigma de la complejidad:

Torbellino describe un movimiento permanente que mantiene la estabilidad de los constituyentes. Es un movimiento a partir del cual se puede desprender la idea de bucle, es decir, la idea de los procesos recursivos, donde cada efecto es a la vez causa y producto [...]; Dialógica, que supone la colaboraciónconflicto como aspectos inseparables de la realidad: se trata de dos principios heterogéneos que están en conflicto y no obstante colaboran [...] Holograma; se trata de una imagen en donde cada punto contiene la información de todo el objeto. [...] lo que quiere decir que no sólo parte está en el todo, sino que el todo está en la parte (pág. 65).

En el pensamiento complejo a través de la dialógica coexisten elementos que pueden ser antagónicos, es decir los asocia siendo al mismo tiempo opuestos y complementarios, el torbellino explica que la consecuencia se vuelve causa en cualquier fenómeno y la causa se vuelve consecuencia; 
considerando la metáfora del holograma en la realidad se puede contemplar lo específico, lo fragmentado sin perder la vinculación con la totalidad.

Es necesario recordar a Morin (2007): quien denomina estos términos como los principios y advierte que ayudan a pensar la complejidad, afirma que el principio dialógico permite mantener la dualidad en el seno de la unidad:

La idea recursiva es, entonces, una idea que rompe con la idea lineal de causa/efecto [...] porque todo lo que es producido reentra sobre aquello que lo ha producido en un ciclo en sí mismo autoconstitutivo, auto-organizador, y autoproductor [...] podemos enriquecer al conocimiento de las partes por el todo y del todo por las partes, en un mismo movimiento productor de conocimientos (pág. 107).

La interdisciplinariedad como modalidad de investigación permite profundizar los niveles de aprehensión y abordaje del objeto de estudio para su comprensión con una visión amplia, significativa, enriquecedora con tantos aportes para la solución de un problema como disciplinas intervengan. Morin (2002): señala que "ínter-disciplina puede querer decir también intercambio y cooperación, lo que hace que la ínter-disciplina pueda convertirse en algo orgánico" (pág. 126).

Esta modalidad permite visualizar realidades de manera sistémica considerando los elementos emergentes, para obtener una concepción que traspase los límites del ámbito del conocimiento micro, con visión reducida de la realidad hacia escalas de estadio superior para aportar aspectos universales o planetarios, estableciendo nexos entre disciplinas no comunicantes.

Por lo tanto, se busca comprender aquellas realidades sociales que para su abordaje necesitan su exposición desde varios ejes multifactoriales, debido a la complejidad que le es propia, es decir, requiere de varias orientaciones disciplinares para su aprehensión, una visión polinuclear, ver los diferentes aspectos de un fenómeno. La dialéctica enriquece y complementa la percepción que se tenga de la realidad ya que a través de la comprensión 
de las diferentes perspectivas que aporta cada disciplina se puede alcanzar a la construcción de nuevas realidades.

La complejidad conduce a la creación de un hombre reflexivo consigo mismo y con su ambiente, del cual está íntimamente ligado, fusionado (el hombre es un sujeto social, es lo que le da sentido), pensar bajo este paradigma le da apertura a una visión más amplia, más integral, el hombre aprehende la realidad, paseándose entre la simplicidad y el holismo, considerando la incertidumbre. Implica tener visión desde varias perspectivas, involucra varias disciplinas, las que a su vez articula, esto debido a los multidimensionales eventos que pueden intervenir.

De aquí surge la teoría de implementar la noción de interdisciplinariedad a fin de favorecer a la solución de los problemas sociales desde el seno de las instituciones educativas habida cuenta que desde cada una de las disciplinas por separado que se ofertan es difícil comprender los problemas globales por el carácter sistémico de la realidad.

La importancia radica en la contribución que cada miembro aporte en pro de una meta común en el proceso de investigación, afianzando un continuum comunicativo, de diálogo y pensamiento crítico para así confluir diferentes puntos de vista. En la praxis el entorno social otorga significado a cada problema que se da en el mismo, la intervención interdisciplinaria debe hacerse bajo el enfoque de investigación acción, de aprender haciendo, de aprendizaje significativo, para superar la dicotomía entre teoría y práctica.

Significa que quienes propician la interdisciplinariedad son investigadores con competencias reconocidas en su área de conocimiento y procura adentrarse en otras áreas, por esta razón se menciona la pluricompetencia debido a la articulación, intercambio y cooperación de diversos conocimientos (disciplinas) para concebir la unidad, para explicar la realidad compleja. Implica apropiarse de lenguaje, conceptos y nuevos métodos para conocer sin renunciar al propio, ese es el reto. 
Debido a la cualidad compleja de la realidad circundante, la visión amplia desde dos o más disciplinas implica una comprensión sistémica, plural, holística, integradora que supera lo unidimensional, lo unilateral, aquellas acciones que favorecen la reducción para aprehender el todo, para explicarlo y para manipularlo; su resultante es el afianzamiento de varias disciplinas a fin de generar las respuestas a los problemas sociales considerando todos los elementos que pueden estar incidiendo con una perspectiva profunda de su análisis, se representa lo social considerando la universalidad y la diversidad.

Implica que para dar respuestas a los grandes problemas que aquejan al hombre en su condición de ser espiritual, social, biológico se considera un cambio de abordaje de sus ejes problemáticos que permita describir su realidad, desde el entorno en el que se desenvuelve hasta un ámbito planetario. Para esto, además de la interdisciplinariedad y en un estadio superior se encuentra el modelo de la transdisciplinariedad que refiere el abordaje de la realidad a través de un cambio de paradigma que trascienda la unidad de y las fronteras de cada disciplina y genere un metalenguaje imbricando dos o más áreas de conocimiento de diferentes disciplinas.

Para transitar hacia la interdisciplinariedad es importante tener una actitud intelectual para abordar la realidad, Ander-Egg (1999i): establece que son dimensiones sustantivas: sistémico: todo unitario y organizado por su naturaleza constituye un todo organizado; ecológico: nada ocurre de modo aislado, todo tiene un acondicionamiento; dialéctico: las partes de la realidad están en un proceso causado por el choque de contrarios y de múltiples factores que se entrecruzan.

Extrapoló estas categorías al ámbito educativo, refiriendo que lo sistémico: cada fenómeno educativo que se analiza debe ser considerado como un aspecto de la totalidad de la que forma parte; lo ecológico significa que debe que el tratamiento de un problema debe considerar efectos, interacciones y retroalimentaciones que existen entre los diferentes 
subsistemas; dialéctico: superación de la dicotomía entre teoría y práctica, un enfoque holístico, pensamiento crítico, función desmitificadora para quitar los velos que encubren la realidad, perspectiva utópica para intervenir el futuro.

La actitud interdisciplinar según Ander-Egg (1999j): implica tener una apertura mental para evitar sesgar la comprensión de la realidad desde la perspectiva de cada profesional, desde el análisis de realidades desde la disciplina de cada uno quitándose las anteojeras del propio campo profesional. Para ello hay que abrirse a nuevas y diversas perspectivas y así evitar el parasitismo mental, siendo que cada fenómeno está condicionado por policausalidad de otros fenómenos en los que estamos insertos.

El enfoque sistémico es útil para interpretar la realidad desde la óptica totalizante $\mathrm{u}$ holista. Todos los elementos que la componen están intrínsecamente relacionados entre sí, caracterizados como sistemas abiertos, los cuales manifiestan cambios en su comportamiento cuando se generan modificaciones en su proceso y en su medio ambiente. Estudia el entorno como punto de partida para analizar y describir su funcionamiento e interacción respecto a este. Busca interpretar similitud de propiedades en diferentes campos, aunque sean intrínsecamente distintos entre sí.

Su concepción se debe a Bertalanffy (1968): quien aborda la comprensión de realidades complejas, en la cual se dan ciertos procesos en permanente interacción con su entorno. Este autor define los sistemas abiertos "un sistema abierto es definido como sistema que intercambia materia con el medio circundante, que exhibe importación y exportación, constitución y degradación de sus componentes materiales" (pág. 146).

Los sistemas abiertos están definidos e influenciados en función a su entorno, lo que conduce a manifestar que pasan de un estado a otro en virtud de esta condición, se construye, alimenta, cambia en función a las acciones ejercidas en su exterior ya que está en constante interacción con sus elementos circundantes. 
Esta propuesta permitirá a la comunidad educativa fortalecerse para una perspectiva diferente de una situación problemática, ya que cada elemento que participa desde cada disciplina tiene un valor y un significado cuando está en interacción unos con otros, es decir, cuando se tiene una visión sistémica. En ese marco, Martínez (1997b): declara respecto al modelo interdisciplinar "funciona como un medio de solucionar problemas "prácticos" dentro de la sociedad" (pág. 110). Para este autor es necesario respetar cada disciplina, converger en un tema específico y en los métodos a través de un plan de acción, así como en los resultados, hasta alcanzar la unidad, basado en el principio de complementariedad que manifiesta que la descripción que se realice de cualquier ente se enriquece con los aportes de diferentes personas, filosofías, métodos y disciplinas.

En este sentido, Del Pozo (1996): deduce que las organizaciones deben ser gerenciadas con la óptica sistémica, lo que equivale a visualizarla en su totalidad y no de manera parcelada en cuanto a las funciones primigenias: planificación, organización, dirección y control, evitando de esta manera, su estudio y análisis a la luz particular de la postura de cualquier miembro del sistema.

Es importante la información (a través de la realimentación) que debe ostentar el gerente, es decir, el epicentro que facilita las decisiones es el acceso que se tiene al conocimiento de la información ya que este aspecto se considera como un mecanismo de relación que facilita la toma de decisiones a fin de considerar estatus real y la evolución organizacional propia del contacto que se tiene con el medio ambiente.

El elemento integrador, que considere los componentes del sistema y el entorno en el cual esta es actor es la sistematización de este, a través de la información que se le suministre a quien realice actividades de gerencia, en este caso educativo, es la que coadyuva a que la organización evolucione y a su vez transfiera los aspectos que favorezcan el cambio necesario en los 
escenarios donde hace vida, siendo la expresión manifiesta el fin de la organización. Es el modelo para una organización cambiante, que se transforma desde su praxis considerando un medio dinámico, con modificaciones recíprocas.

La interrelación entre cada uno de los integrantes de la organización es importante considerarla, ya que la actuación u omisión vista de manera individual afecta de manera consecuente a otros. La entrada, procesamiento, salida y la realimentación de los procesos es importante, siempre que se conduzca con el enfoque integracionista.

El pensamiento sistémico organiza unidades en círculos de relaciones causa efecto o flujos recíprocos en el que toda influencia es causa y efecto, es inexistente la influencia en una sola dirección (realimentación). Desde esta perspectiva se considera que el ser humano forma parte del sistema sin estar separado de él, de allí que todo agente participante se hace responsable del éxito o de cualquier problema que se genere en el mismo.

Acorde a lo planteado en el enfoque sistémico e interrelacionado con la temática que se está abordando en este ensayo, Ortiz (2012), afirma respecto a los beneficios de la interdisciplinariedad en la educación que:

[...] Permite una visión integral del objeto de estudio, estimula la aparición de nuevas concepciones teóricas y metodológicas novedosas para la solución de los problemas científicos y contribuye a elevar el potencial teórico de las ciencias, y por tanto, incrementa su pertinencia ante las crecientes demandas del desarrollo social (pág. 7).

En referencia al tema, Ander-Egg (1999k): propone un esquema para la realización de la interdisciplinariedad en educación: a). Formular un marco referencial: ubicar un tema delimitado y concreto; b). Trabajar acorde a un esquema de investigación acción: se realiza un diagnóstico de problemas de índole social, proponiendo alternativas para mejorar la situación aplicando conocimientos y resultados del trabajo interdisciplinar; c). Explicitar y 
programar las actividades que se han de realizar: surgiendo del marco de referencia; d). Elaboración y presentación del trabajo final

La postura del autor Ander-Egg (1999l): demuestra la importancia que tiene para los investigadores enmarcados en el paradigma de la interdisciplinariedad el acoplamiento de un diseño de una guía de acción a manera de sistematizar las acciones investigativas que a bien tienen razón de hacer, esto con la participación de docentes y de estudiantes, el proyecto se enriquece con el aporte de multiplicidad de actores.

El autor mencionado considera que se hace necesario que existan condiciones para hacer de la interdisciplinariedad una práctica educativa: formación docente, interiorización de la práctica interdisciplinaria, interés de parte del docente, motivación del estudiantado, elaboración de un marco referencial que articule aspectos fragmentarios y encuadre la estrategia pedagógica, elección del tema de carácter pedagógico, integración de asignaturas que aporten al objeto de estudio, lectura y discusión del marco referencial, perfilar los estudiantes que se integrarán, realización de trabajos para la presentación de resultados, presentación del tema.

El ensayista incorpora dentro de la obra las condiciones que deben darse para la incorporación de la interdisciplinariedad en la educación, la misma parte de la disciplina que posee cada profesor y de la cual debe tener dominio para presentar su postura ante el análisis de un determinado problema, es decir desde la óptica de su materia o unidad curricular que asiste, es importante que la escogencia de la temática que no admita una visión disciplinar o reduccionista, debe desarrollarse en función a la diversidad de posturas de los docentes y estudiantes que participan, la importancia de la documentación por parte de todos y someterse a la rigurosidad que exige la presentación del informe que contenga los aspectos abordados en la investigación.

Por lo expuesto anteriormente la resolución de problemas complejos 
debe hacerse a partir de distintas formas de pensar, buscando marcos integradores que favorezcan la comprensión de fenómenos para procurar solventar escenarios discontinuos.

Por lo tanto, reseña Ander-Egg (1999m): que las exigencias para la práctica de la interdisciplinariedad propicia el trabajo en equipo; disposición al diálogo y desarrollo del pensamiento complementario; tener una perspectiva globalizadora de la realidad; transitar del paradigma de la simplificación al paradigma de la complejidad; integrar el trabajo interdisciplinario entre docentes y alumnos un buen clima de interacción pedagógica; avanzar hacia la construcción de un lenguaje interdisciplinario e integración de principios epistemológicos comunes; cada docente debe mantener el interés por saber qué hacen los otros profesores.

Para alcanzar la Interdisciplinariedad en Educación se debe tener un pensamiento que tenga como epicentro de acción el paradigma complejo, para comprender los diferentes aspectos de la realidad social, transitar de la disertación del objeto de estudio desde la fragmentación de saberes hacia la comprensión de problemas globales. El enfoque debe ser por lo tanto sistémico, ecológico y dialéctico, con una actitud existencial como modo de abrirse al mundo exterior.

Demanda en educación apropiarse de un esquema de acción para la generación de investigaciones bajo la modalidad investigación acción donde se considere la integración de profesores y de estudiantes, desde el abordaje de realidades hasta la presentación de informe con los hallazgos para solventar problemas sociales.

\section{Conclusiones}

A través de la interdisciplinariedad y del pensamiento complejo se permite tener un reconocimiento y puntos de convergencia entre las diferentes disciplinas para solventar los problemas que aquejan la sociedad, puesto que 
desde el ámbito educativo emanan las respuestas, condicionando desde su seno su praxis. Se hace necesario realizar cambios a nivel institucional, visualizarse entre cada unidad curricular, sin fronteras que le separen, para lo cual se deben gestar modificaciones a nivel curricular, fomentando y sensibilizando este nuevo enfoque de hacer ciencia a estudiantes y docentes.

La Interdisciplinariedad en Educación implica una nueva modalidad de pensamiento en la academia, en la que se visualiza el entorno desde la óptica de cada docente asumiendo en primera instancia su postura desde su ámbito de acción o disciplina, el abordaje de realidades. La interdisciplinariedad trasciende la disciplinariedad en virtud de la complementariedad entre diferentes ámbitos del saber, aportando conocimientos desde una visión holística, integradora y participativa.

El estudio de la Interdisciplinariedad es de gran relevancia para el mundo académico, ya que aporta ideas para la generación de un cambio en el sector educativo en tanto se logre una transformación del pensamiento entre todos los actores involucrados, para que desde una visión holística de las realidades, se pueda dar soluciones a las problemáticas del entorno educativo, lo que se traduce en instituciones transformadas en epicentros de conocimiento socializado, transformador de realidades, enriqueciendo los procesos y aprendiendo la interdisciplinariedad en cada caso particular.

\section{Referencias}

Ander-Egg E. (1999a,b,c,d,e,f,g,h,i,k,l,m). Interdisciplinariedad en Educación. 3ra edición, ISBN: 950-550-125-0. Colección Respuestas Educativas. Argentina: Editorial Magisterio del Río de la Plata.

Balza, A. (2011). Complejidad, Transdisciplinariedad y Transcomplejidad:

Los Caminos de la Nueva Ciencia. ISBN: 978-890-12-4780-7. Venezuela: Fondo Editorial Gremial. Asociación de Profesores Universidad Nacional Experimental "Simón Rodríguez" (APUNESR). 
Bertalanffy, L. (1968). Teoría General de los Sistemas: Fundamentos,

Desarrollo, Aplicaciones. Primera edición, ISBN: 968-16-0627-2. México: Fondo de Cultura Económica.

Del Pozo, F. (1996). La Dirección por Sistemas. ISBN: 968-18-0579-8.

México, D.F.: Editorial Limusa, S.A. de C.V.; Grupo Noriega Editores.

Martínez, M. (1997a,b). El Paradigma Emergente: Hacia una Nueva Teoría

de la Racionalidad Científica. 2da. Edición, ISBN: 968-24-0415-0. México: Editorial Trillas.

Morin, E. (2002). La Cabeza Bien Puesta. Repensar la Reforma. Reformar

el Pensamiento. 1ra edición, 5ta reimpresión, ISBN: 950-602-395-6.

Buenos Aires, Argentina: Editorial Nueva Visión.

Morin, E. (2007). Introducción al Pensamiento Complejo. ISBN: 978-847432-518-8. Barcelona, España: Editorial Gedisa.

Ortiz, E. (2012). La interdisciplinariedad en las investigaciones educativas.Didasc@lia: Didáctica y Educación, 3(1), 1-12, e-ISSN: 2224-2643. Recuperado de:

https://dialnet.unirioja.es/servlet/articulo?codigo $=4228305$

Prigogine, I. \& Stengers, I. (1990). La Nueva Alianza: Metamorfosis de la Ciencia. Segunda edición, ISBN: 84-206-2368-7. Madrid, España: Alianza Editorial, S.A. 


\section{Ensayo Original / Original Essay}

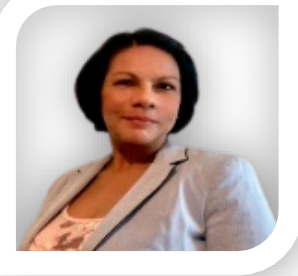

\section{Lisbeth Adays RengifoAvadez \\ e-mail: lisbethrengifo@gmail.com}

Nacida en Caracas, capital de Venezuela, el 15 de diciembre del año 1971. Doctora en Educación por la Universidad Pedagógica Experimental Libertador (UPEL); Magister Scientiarum en Ciencias de la Educación por la Universidad Santa María (USM); Especialista en Docencia Universitaria, Universidad Santa María (USM). Especialista Gerencia Empresarial por la Universidad Santa María; Licenciada en Administración de la Universidad Santa María; Docente Adscrita a la Universidad Nacional Experimental Politécnica de la Fuerza Armada (UNEFA), desde el año 2006 hasta la actualidad; Docente de Administración de Recursos Humanos, Principios de Gerencia, Principios de Administración; Desarrollo actividades investigativas como responsable de la Línea de Investigación en Estado, Gerencia y Sociedad.

El contenido de este manuscrito se difunde bajo una Licencia de Creative Commons ReconocimientoNoComercial-Compartirlgual 4.0 Internacional 